doi: $10.1016 / j$. fct.2006.06.020

Copyright (c) 2006 ILSI Europe Published by Elsevier Ltd.

\title{
Risk assessment of substances that are both genotoxic and carcinogenic
}

\section{Report of an International Conference organized by EFSA and WHO with support of ILSI Europe}

\section{S. Barlow ${ }^{a}$, A.G. Renwick', J. Kleinerc, J.W. Bridges ${ }^{d}$, L. Busk ${ }^{e}$, E. Dybingf, L. Edlerg, G. Eisenbrand ${ }^{h}$, J. Fink-Gremmels', A. Knaap ${ }^{j}$, R. Kroes $^{k}$, D. Liem $^{\text {c }}$, D.J.G. Müller', S. Page ${ }^{m}$, V. Rolland ${ }^{\mathrm{c}}$, J. Schlatter ${ }^{\mathrm{n}}$, A. Tritscher $^{\mathrm{m}}$, W. Tueting $^{\circ}$ and G. Würtzen ${ }^{p}$}

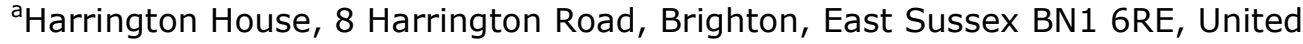 \\ Kingdom \\ bUniversity of Southampton, School of Medicine, Biomedical Sciences Building, \\ Bassett Crescent East, SO16 7PX Southampton, United Kingdom

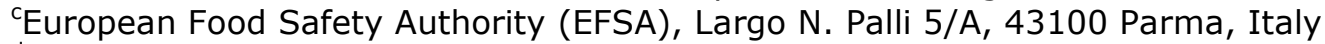 \\ dUniversity of Surrey, School of Biomedical and Life Sciences, Guildford GU33 AE, \\ Surrey, United Kingdom \\ ${ }^{\mathrm{e}}$ National Food Administration, Research and Development Department, P.O. Box \\ 621, 75126 Uppsala, Sweden \\ ${ }^{f}$ Norwegian Institute of Public Health, Division of Environmental Medicine, P.O. \\ Box 4404, Nydalen, 0403 Oslo, Norway \\ ${ }^{9}$ German Cancer Research Centre (DKFZ), Department of Biostatistics C060, Im \\ Neuenheimer Feld 280, 69120 Heidelberg, Germany \\ hUniversity of Kaiserslautern, Department of Chemistry, Food Chemistry and \\ Environmental Toxicology, P.O. Box 3049, 67653 Kaiserslautern, Germany \\ 'Utrecht University, Faculty of Veterinary Medicine, Department of Veterinary \\ Pharmacology, Yalelaan 16, P.O. Box 80152, 3508 TD Utrecht, The Netherlands \\ ${ }^{j}$ National Institute of Public Health and the Environment (RIVM), Centre for \\ Substances and Risk Assessment, A. van Leeuwenhoeklaan 9, P.O. Box 1, 3720 \\ BA Bilthoven, The Netherlands \\ kUtrecht University, Institute for Risk Assessment Sciences (IRAS), Faculty of \\ Veterinary Medicine, Yalelaan 2, P.O. Box 80176, 3508 TD Utrecht, The \\ Netherlands \\ 'Procter \& Gamble Service GmbH, Sulzbacher Str. 40, 65824 Schwalbach am \\ Taunus, Germany \\ morld Health Organization (WHO), International Programme on Chemical Safety, \\ Avenue Appia 20, 1211 Geneva 27, Switzerland \\ ${ }^{\mathrm{n}}$ Swiss Federal Office of Public Health, Food Toxicology Section, \\ Stauffacherstrasse 101, 8004 Zurich, Switzerland \\ ${ }^{\circ}$ International Life Sciences Institute, European Branch (ILSI Europe), Avenue E. \\ Mounier 83/Box 6, 1200 Brussels, Belgium \\ ${ }^{\mathrm{P} C o c a-C o l a ~ N o r d i c ~ a n d ~ G e r m a n y ~ D i v i s i o n, ~ S t r a n d v e j e n, ~ 60, ~} 2900$ Hellerup, \\ Denmark
}

Received 19 June 2006; accepted 26 June 2006. Available online 8 July 2006.

\section{Abstract}

The European Food Safety Authority (EFSA) and the World Health Organization (WHO), with the support of the International Life Sciences Institute, European 
Branch (ILSI Europe), organized an international conference on 16-18 November 2005 to discuss how regulatory and advisory bodies evaluate the potential risks of the presence in food of substances that are both genotoxic and carcinogenic. The objectives of the conference were to discuss the possible approaches for risk assessment of such substances, how the approaches may be interpreted and whether they meet the needs of risk managers. ALARA (as low as reasonably achievable) provides advice based solely on hazard identification and does not take into account either potency or human exposure. The use of quantitative lowdose extrapolation of dose-response data from an animal bioassay raises numerous scientific uncertainties related to the selection of mathematical models and extrapolation down to levels of human exposure. There was consensus that the margin of exposure (MOE) was the preferred approach because it is based on the available animal dose-response data, without extrapolation, and on human exposures. The MOE can be used for prioritisation of risk management actions but the conference recognised that it is difficult to interpret it in terms of health risk.

Keywords: Margin of exposure; Genotoxic; Carcinogenic; Risk assessment

\section{Introduction}

One of the most difficult issues in food safety is to advise on potential risks for human health when it is found that compounds which are both genotoxic and carcinogenic are present in food and their presence cannot be readily eliminated or avoided.

Currently, there are various approaches used to assess the risks from substances that are genotoxic and carcinogenic. The advice offered depends not only on the technical approach adopted by the regulatory or advisory body providing the advice, but also on the legal background in the region or country concerned.

The term genotoxic is used if a substance or its active metabolite affects cellular DNA. Normal cells develop into cancerous cells by the loss of genomic stability and sequential acquisition of genetic alterations (Gray and Collins, 2000 and Loeb and Loeb, 2000). Carcinogenesis is a multistep process that can be affected by chemicals in a variety of ways.

Carcinogenic processes can be categorised in two major modes of action:

(i) The first is a DNA-reactive mode of action whereby the compound or its active metabolite reacts covalently with DNA in target cells of carcinogenicity to cause pre-carcinogenic mutations, which lead to neoplastic transformation and neoplasm induction. For this mode of action it is considered that there may be no threshold in the dose-response relationship and therefore there is no dose without a potential effect.

(ii) The second mode of action is epigenetic in nature and does not involve chemical reactivity and covalent binding to DNA. In this case the compound produces effects in target cells of carcinogenicity that either indirectly lead to neoplastic transformation or facilitate the development of neoplasms from cryptogenically transformed cells. For these processes it is generally assumed that there is a level of exposure below which no significant effect will be induced (Dybing et al., 2002). That means that homeostatic mechanisms would be able to counteract any perturbation produced by low levels of intake, and that structural 
or functional changes leading to adverse effects, including cancer, would be observed only at higher intakes. Examples are compounds that affect spindle function or chromosome integrity, or cause indirect damage to DNA.

Historically, different approaches have been adopted for the risk characterisation of threshold and non-threshold effects (WHO, 1999). For threshold-effects it is current practice to estimate the level of exposure without significant adverse effect and to derive a health-based guidance value such as a tolerable daily intake. Such an approach would not be used for DNA-reactive substances which are both genotoxic and carcinogenic, as it is assumed that there is no exposure without any potential effect, i.e. it is suggested that exposure to even a single molecule could produce DNA damage. On the other hand, the presence of a DNA adduct does not in itself have genetic consequences. It needs to be fixed into a mutation through DNA replication and is dependent on the rates of DNA repair and cell proliferation (Lutz, 1990). Since there is currently no international scientific consensus on what is the best approach for assessing the risk for substances that are both genotoxic and carcinogenic, the advantages and disadvantages of various approaches were discussed at the joint EFSA/WHO/ILSI Europe conference, based on three papers developed by these organizations.

As outlined above, the classification of a substance as either possessing or not possessing DNA-reactive, genotoxic and carcinogenic properties has major consequences for the risk assessment approach to be taken and the advice to be given to risk managers. In the hazard identification step this decision is made based on a weight of evidence approach and two critical questions need to be answered:

1. What is the evidence that the compound is a DNA-reactive genotoxicant?

2. What is the evidence that the compound is a carcinogen?

To answer the first question, information from various sources needs to be considered (none of which may be conclusive alone) and the overall decision is mostly made on a weight of evidence approach using expert judgement. The main criteria for the decision on the mode of action are:

- Is the compound genotoxic in standard in vitro and/or in vivo genotoxicity assays?

- Is there evidence of direct interaction with DNA via DNA-reactivity?

- Is the compound structurally related to other known DNA-reactive substances that are both genotoxic and carcinogenic?

The second question, on the evidence of carcinogenicity, may be complex particularly if the data set is incomplete. The decision is often based on a standard two-year carcinogenicity bioassay in rodents but other data can provide the basis for, or contribute to, the overall conclusions (weight of evidence approach), e.g. are adequate epidemiological data available, is the compound carcinogenic in alternative cancer assays or does the compound induce preneoplastic lesions, such a altered hepatic foci?

In cases where a substance has been shown to be carcinogenic but its carcinogenic mode of action has not been identified, the default assumption is that genotoxicity is the mode of action leading to cancer. 
Against this background, the conference addressed the following questions:

- What possibilities are there for risk assessment for genotoxic and carcinogenic substances ingested at low intakes?

- How should the outcomes of the various approaches be interpreted in terms of risks to human health?

- To what extent do the approaches available meet the needs of risk managers?

- Are the approaches helpful in giving practical options for risk managers in situations where exposure cannot be completely eliminated and the magnitude of risk cannot be readily determined?

This report summarises the presentations and discussions from the conference. It identifies aspects on which there was general consensus and outlines the proposals made for further development of a common approach.

\section{Approaches proposed by EFSA, WHO and ILSI Europe}

\subsection{Summary of the background documents}

Three documents were provided as the basis for discussions at the conference:

(i) ILSI Europe Expert Group draft paper on Approaches to the Risk Assessment of Genotoxic Carcinogens in Food: A Critical Appraisal (O'Brien et al., 2006).

(ii) Opinion of the EFSA Scientific Committee related to a Harmonised Approach for Risk Assessment of Substances which are both Genotoxic and Carcinogenic (EFSA, 2005).

(iii) Summary and Conclusions of the 64th Meeting of the Joint FAO/WHO Expert Committee on Food Additives (JECFA, 2005), which included advice on compounds that are both genotoxic and carcinogenic.

They considered similar scientific data and reached similar overall conclusions. The aims and objectives for the activities differed and this was reflected in the nature of their final recommendations.

The ILSI Europe Expert Group was established to review and analyse the approaches currently applied by risk assessors to genotoxic carcinogens (substances that are both genotoxic and carcinogenic), and to undertake a critical assessment of the scientific basis of the mechanism of action and dose-response relationships. The overall aim was to produce guidance for risk assessors working in the food industry when low levels of genotoxic carcinogens are detected as contaminants in food. The paper reviews the types of hazard identification data that would lead to conclusions that a chemical is a DNA-reactive genotoxicant and/or that a chemical is a carcinogen. Under hazard characterisation, the paper reviews both toxicokinetic and toxicodynamic factors that could influence the production of cancer and the linearity of the overall dose-response relationship. Risk characterisation focused on four different approaches:

(i) ALARA (reduce exposure to as low as reasonably achievable),

(ii) low-dose extrapolation of data from rodent carcinogenicity bioassays, 
(iii) TTC (threshold of toxicological concern) for substances that are genotoxic and for which adequate cancer dose-response data are not available, and

(iv) margin of exposure (MOE), which is the ratio between a reference point on the dose-response data from experimental or epidemiological studies and the estimated human exposure.

The EFSA Opinion provided a summary review of the biological processes leading to cancer and the problems of extrapolation of cancer dose-response data. The Opinion then discussed the data requirements for derivation of an MOE, i.e. the point selected on the dose-response curve and the measure(s) of intakes that would be most suitable for the formulation of advice to risk managers. The Opinion then provided guidance on the interpretation of the calculated MOE and the sources of uncertainty and variability that could influence perceptions of the magnitude of the numerical estimate. The Opinion gave basic guidance on how the MOE should be calculated and advice on the magnitude of a value that would indicate a low concern from a public health point of view and that might be considered a low priority for risk management actions.

The 64th JECFA addressed in the General Considerations section of the report the limitations of advice based on the ALARA principle for risk management purposes, since it does not take human exposure or carcinogenic potency into account. The problems associated with the formulation of scientifically sound advice for risk managers on substances that are both genotoxic and carcinogenic was also discussed. The JECFA considered three approaches: the MOE, extrapolation of the dose-response curve outside the observed dose range and linear low-dose extrapolation from a point of departure. The Committee concluded that MOE and linear extrapolation were the only approaches practicable at present, and that MOE was preferred because it does not give a numerical risk estimate that may be regarded as quantification of the actual risk. The Committee applied this approach to the risk assessments of three genotoxic carcinogens, acrylamide, ethyl carbamate and polycyclic aromatic hydrocarbons, which are present as contaminants in food. Margins of exposure (MOEs) were calculated for each substance for different exposure conditions, considering mean and high-level consumer exposure.

\subsection{Similarities in the three approaches}

All three papers reached a number of similar conclusions:

(i) The traditional approach of using a no-observed-adverse-effect level (NOAEL) to define a health-based guidance value, such as a tolerable daily intake, is not appropriate for DNA-reactive substances that are both genotoxic and carcinogenic. Although a biological threshold below which cancer is not induced may exist for such substances, the position of the threshold could not be inferred from the position of the no-observed-effect level (NOEL) on a dose-response curve.

(ii) The MOE is the most scientifically credible approach to the formulation of advice because it takes account of intake/exposure and the available data on the dose-response relationship are used without extrapolation or the generation of possibly uncertain risk estimates.

(iii) The point on the cancer dose-response curve used in the MOE calculation should be close to the bottom end of the observed effect range or limit of quantification. A $10 \%$ increased tumour incidence over background was chosen as 
the benchmark response (BMR) in the EFSA Opinion and the JECFA Report. Although other response levels could be scientifically valid, such as $5 \%$ or $20 \%$, a single general recommendation was proposed because one of the uses of the MOE is for comparisons between different substances.

(iv) Mathematical modelling is used to define the benchmark dose (BMD) giving the benchmark response (IPCS, 2004). The use of the lower limit of the confidence interval on the BMD (the BMDL) was recommended because this reflected uncertainties and statistical errors in the available cancer dose-response data. The T25 (Dybing et al., 1997) could be used in cases where the doseresponse data were not adequate to define the BMD or $B M D L$, but the use of the T25 should be clearly pointed out in the advice to the risk manager and would affect the interpretation of the MOE in relation to human health.

(v) Exposure/intake assessment of substances that are both genotoxic and carcinogenic is not inherently different to that of other chemicals to which humans are exposed via the diet. Intake estimates should be provided for various percentiles of the general population, such as the 50th, 90th, 95th and 97.5th percentiles, and for any subgroups with higher intake patterns. Different intakes will result in a range of MOE values for the same substance; such information should be useful in the development of risk management strategies.

(vi) Risk managers should be given advice to aid them in the interpretation of the magnitude of estimated MOE in relation to human health. Without such advice an MOE would be of little more value than ALARA (that is until sufficient substances had been evaluated to produce a ranking based on their MOE values).

\subsection{Differences between the three documents}

The documents differed in various respects:

(i) The amount of guidance given on methods to calculate the BMD and BMDL varied greatly. The most detailed advice was in Annex 1 of the JECFA Report, which listed 8 different models plus an approach to the assessment of goodness of fit.

(ii) The uncertainties that would have to be allowed for before any MOE could be considered to be of low concern from the public health point of view was not considered in the ILSI Europe paper, because the information is used at the interface between risk assessors and risk managers. This issue was considered most comprehensively in the EFSA Opinion, which concluded that a MOE of 10,000 and above, based on a BMDL10 from an animal study, would be a value that would indicate a low concern from a public health point of view and that might be considered a low priority for risk management actions. The rationale for this value was twofold. A 100 -fold difference between the BMDL and human exposure would be necessary to take into account general issues of species differences and human variability (analogous to the use of a 100-fold uncertainty factor for threshold toxicants). A further 100 -fold difference would be necessary because of additional uncertainties related to human variability in cell cycle control and DNA repair, and because the shape of the dose-response curve below the BMD and the dose level below which the cancer incidence is not increased are unknown. ILSI Europe and JECFA applied the proposed MOE approach to the risk assessment of specific food contaminants, whereas the EFSA Opinion developed an approach for future application by EFSA Panels. 
(iii) The assessment of acrylamide, ethyl carbamate and polycyclic aromatic hydrocarbons in the JECFA Report gave advice that is compatible with the conclusion of the EFSA Opinion, i.e. that an MOE of 10,000 or above indicates low public health concern. For acrylamide the JECFA concluded that the MOEs were low (the MOEs were 300 for the average intake and 75 for intakes by high consumers) and indicated a human health concern. For ethyl carbamate the intakes from foods excluding alcoholic beverages were of low concern because the MOE was 20,000, whereas intakes from foods including alcoholic beverages were of concern because the MOE was 3,800. The JECFA concluded that the intakes of polycyclic aromatic hydrocarbons were of low concern for human health, since the MOEs for average and high consumers were 25,000 and 10,000, respectively.

(iv) The ILSI Europe paper presented MOE values for acrylamide, aflatoxin-B1, benzo(a)pyrene, dimethylnitrosamine, ethyl carbamate and phenyl imidazopyridine, and concluded that the values could be used to prioritise risk management actions.

\subsection{Aspects not covered in the documents}

A number of important aspects not covered or not addressed in detail in the documents were identified in the conference presentation by Diane Benford.

(i) There should be an agreed strategy for assessing genotoxicity to aid harmonisation of the MOE approach.

(ii) Approaches are needed for genotoxic chemicals for which it is not possible to identify a BMDL or T25, either because there are too few cancer data, or because there is a high incidence of tumours at all tested doses.

(iii) There needs to be discussion on whether MOEs would be useful in the risk assessment of proven human carcinogens.

(iv) The MOE approach does not preclude the application of ALARA to the same substance. For example, if the intake of a substance that is both genotoxic and carcinogenic can be reduced readily, then this should be considered by risk managers irrespective of the calculated MOE.

(v) The types of substances for which the MOE approach would be applied need to be discussed by risk managers and stated more clearly. It should be stated that a large MOE is not a basis for approving the deliberate addition or use of genotoxic chemicals in food, for condoning infringements of regulations (such as the case of Sudan 1 in 2005), or for relaxing standards.

(vi) All three documents considered the uncertainties associated with the doseresponse characterisation but did not adequately recognise that uncertainties in the intake estimates are of equal importance in the numerical value of the MOE. This is an important difference between an MOE and a health-based guidance value, such as a TDI, where the errors in the value are derived solely from the dose-response data and their interpretation.

\section{Discussions and outcome of the conference}

The conference began with presentations on the three key documents and the evolution of the underlying science. Parallel working groups of participants then considered issues raised by the different approaches to the assessment of 
compounds that are genotoxic and carcinogenic (see Section 1). The report that follows summarises the output from the working groups and the final discussion in plenary session.

\subsection{The ALARA approach}

The conference noted the inherent problems when advice to risk managers on substances that are both genotoxic and carcinogenic is based on the ALARA approach, i.e. that intakes should be as low as reasonably achievable. This approach is closely related to ALARP (as low as reasonably practicable), which is a policy adopted by risk managers to reduce intakes. ALARP acknowledges that there are economic costs in reducing risks that may have to be taken into account. The ALARA approach has been used by several advisory bodies and national regulatory authorities, which consider that other approaches, such as using experimental animal dose-response data as a surrogate for human response to derive a quantitative estimate of risk, can give misleading indications of potential risks to human health at very low exposures.

The ALARA approach has the advantage that only hazard identification data are needed to confirm that the substance is either genotoxic (in vivo) and so assumed to be carcinogenic, or shown to be both genotoxic and carcinogenic. Such data may come from animal and/or human studies, but for most substances human data are rarely available. For risk assessors, the ALARA approach has the practical advantage that there is no requirement to evaluate the carcinogenicity data in a quantitative manner, no mathematical or computing expertise is required, and there is no need to evaluate exposure data. The approach is purely qualitative and readily understood by non-scientists.

The key disadvantages of the ALARA approach are that it does not make best use of all the data that are usually available, and is not useful for comparisons between different compounds or for the establishment of priorities for risk management action. It does not make any distinction between high potency and low potency carcinogens, nor does it relate the potential hazard to the level of exposure (risk characterisation). It does not give any estimate of risk and will not give risk managers sufficient information to assess the degree of urgency and extent of risk reduction measures that may be required. In response to ALARA, risk managers may feel they have little choice but to treat all carcinogens similarly with respect to risk reduction. The ALARA approach can also create problems for risk communication; because ALARA provides only a simple qualitative message about genotoxic carcinogens, it is difficult to convey that some substances may be more hazardous than others, either because of their potency or because of the exposure levels.

\subsection{Methodological options for risk characterisation}

\subsubsection{Review of the available tools for risk characterisation}

The conference discussed the different tools that are currently available for risk characterisation of substances that are both genotoxic and carcinogenic. These included:

- Dose-response modelling of experimental animal data (or human epidemiological data, if available) within the observed dose range.

- Calculation of the margin of exposure (MOE) between a reference point in the observed dose range (e.g. the BMDL10) and estimated human exposure. 
- Linear extrapolation from dose-response modelling to estimate risk for the human population at much lower doses.

- Application of the threshold of toxicological concern (TTC).

- Use of DNA adduct data.

\subsubsection{Dose-response modelling within the observed dose range}

Dose-response assessment of tumour incidences within the observed experimental dose range of an animal carcinogenicity bioassay is necessary for either calculation of an MOE or linear extrapolation to estimate risk at lower doses. The principles of such modelling were presented at the conference by Wout Slob, who emphasised, inter alia, that the outcome of any model is only as good as the data available for input; modelling does not provide an alternative that can compensate for inadequate data.

Dose-response modelling can provide several useful reference points, or so-called 'points of departure'. These are numerical values of dose within or close to the experimental tumorigenic range (or the statistical estimate of that range) which can be used to calculate the MOE, or from which to make an estimate of risk at lower doses by extrapolation. Reference point is the terminology used in descriptions of the MOE approach. Point of departure is the terminology used in descriptions of extrapolation approaches. The various reference points/points of departure that can be used include:

- Benchmark dose (BMD) or the statistical lower bound of its $95 \%$ confidence interval (BMDL).

- Lowest-observed-adverse-effect level (LOAEL).

- Carcinogenic potency estimates, such as $\mathrm{TD}_{50}$ and $\mathrm{T} 25$.

The conference agreed that if there were sufficient data of good quality on a substance, then dose-response modelling should be carried out. Suitable data would include experimental bioassay data from studies in animals and/or human epidemiological data.

\subsubsection{Benchmark dose approach}

There was a consensus at the conference that the benchmark dose (BMD) approach offers the best tool for deriving a reference point/point of departure within the observable dose range. The ready availability of special software for developing BMDs, which was not difficult to use, was noted (US EPA, 2000a and US EPA, 2000b). The BMD approach can be applied when there are as few as two dose groups showing tumours and one control group, but it was noted that others have suggested that a larger number of dose groups, such as three plus controls, may be preferable (Edler et al., 2002).

The BMD approach makes full use of all the data points on the dose-response curve; a mathematical model is fitted to the experimental data within the observable range and the dose that causes a low but measurable response (benchmark response or BMR) is chosen as the BMD. BMDs can be modelled for each tumour type and for overall tumour incidence. Typically, the BMR value chosen is a $5 \%$ or $10 \%$ incidence above the control incidence (US EPA, 1995). 
The BMD lower limit (BMDL) refers to the one-sided lower $95 \%$ confidence limit of the BMD. The BMDL, rather than the BMD as a reference point/point of departure, takes into account the statistical uncertainty inherent in the BMD in any given study. The BMDL will be lower than the corresponding BMD and therefore more conservative. Use of the BMDL also assures, with $95 \%$ confidence, that the chosen BMR is not exceeded. It was noted that the BMD approach takes no account of information on mode of action.

\subsubsection{LOAEL}

It was noted that the LOAEL was an easily identified reference point/point of departure from the raw data, requiring no mathematical modelling of the doseresponse relationship. It could be used for calculation of an $M O E$, but its value will be dependent on the individual study design. Its use did not take account of possible uncertainties in the study data. There was little further discussion of this option.

\subsubsection{Carcinogenic potency estimates}

Carcinogenic potency estimates, such as the $\mathrm{T}_{25}$ and $\mathrm{TD}_{50}$, can also be used as reference points/points of departure. Carcinogenic potency estimates give an indication of the dose of a substance administered over a standard animal lifespan that results in a fixed incidence of tumours, say, $10 \%, 25 \%$ or $50 \%$ for the $\mathrm{BMD}_{10}$ (or $\mathrm{ED}_{10}$ ), $\mathrm{T} 25$ and $\mathrm{TD}_{50}$, respectively, after correction for the spontaneous background incidence of tumours among controls. In common with the benchmark dose approach, carcinogenic potency estimates also make use of all the available dose-response data.

Use of the ED $10 / L D_{10}$ has been favoured by the US EPA, 1996 and US EPA, 1999 and other regulatory bodies as a point of departure to estimate risks at lower doses by linear extrapolation. The T25 method, proposed by Dybing et al. (1997) can be used to compare and rank substances for their carcinogenic potency, to calculate MOEs, or to estimate risks at lower doses by linear extrapolation (Sanner et al., 2001). It was noted that the T25 approach has been used in risk assessment for regulation of non-food, genotoxic and carcinogenic chemicals in the EU. The $T D_{50}$ method was proposed by Peto et al. (1984) and numerical $T_{50} S$ have been elaborated in the Carcinogenic Potency Database for over 700 substances (Gold et al., 1984, Gold et al., 1986, Gold et al., 1987, Gold et al., 1989, Gold et al., 1990, Gold et al., 1991, Gold et al., 1992, Gold et al., 1993, Gold et al., 1995 and Gold et al., 1999). Calculation of a TD 50 requires specific software, whereas the T25 can be more easily calculated without computing the dose-response curve. It was noted that linear extrapolation from the $\mathrm{TD}_{50}$ can seriously over- or underestimate the true risk and that it is more often used only for comparing carcinogenic potencies.

The conference noted that comparison and ranking of carcinogenic potencies do not take into account the level of human exposure.

\subsubsection{Calculation of the MOE}

The margin of exposure (MOE) is obtained by dividing the value of the selected reference point on the dose-response curve for the adverse effect of the substance by the estimated human intake of the substance. Being a ratio, it is a dimensionless number. 
The conference considered that a BMDL is the most appropriate reference point for calculating an MOE. The T25 could also be used to calculate an MOE, but it was noted that the resulting value would require a different interpretation from an MOE derived from a BMDL10 as it is based on a different level of response and is usually derived from fewer data points.

\subsubsection{Linear extrapolation to risk at low doses}

In low-dose, linear extrapolation, mathematical models are fitted to the data on tumour incidence to produce quantitative estimates of risk at exposures several orders of magnitude (usually 4 or more) below the lowest experimental dose point. The models that may be used and their strengths and limitations have been discussed in detail elsewhere (Edler et al., 2002). Linear extrapolation has been used in one form or another by a large number of advisory and regulatory bodies, such as the US EPA, and those in some European countries and elsewhere.

Simple linear extrapolation can be carried out from either a selected incidence within the experimental range (e.g. the LOAEL), or from a derived incidence, such as one of the points of departure described above (e.g. BMDL10 or T25) obtained by fitting a mathematical model to the observed data. Alternatively, a more complex mathematical model may be fitted to the data points to estimate the risk at lower doses. A variety of such models are available. The model most widely used to date has been the linearised multistage (LMS) model for which software is available.

In using these approaches, low risk is often defined, for example, as the level of exposure to a substance associated with an upper bound, lifetime risk of cancer of 1 in a million persons $\left(1 \times 10^{-6}\right)$ or one in a hundred thousand $\left(1 \times 10^{-5}\right)$.

The conference noted that different mathematical models, which provide equally good fits to the experimental animal data, can result in very different estimates of risk at low doses. The conference also noted that the mathematical models tend to be highly conservative (some would say overly conservative), with the one-hit model being the most conservative. Thus linear extrapolation should be considered as giving an upper bound estimate of risk rather than the most likely estimate of risk.

\subsubsection{TTC approach}

The threshold of toxicological concern or TTC approach has been advocated for risk assessment of contaminants in food in cases where the biological data are few but the chemical structure is known and there are good exposure data (Kroes et al., 2004). In this approach, if the substance is genotoxic or has a structural alert for genotoxicity and does not belong to a group of identified structures that are likely to be the most potent genotoxic carcinogens, exposures below $0.15 \mu \mathrm{g} /$ day (or $0.0025 \mu \mathrm{g} / \mathrm{kg}$ bw/day) are considered to be of negligible risk. This figure is based on linear extrapolation of bioassay data for substances having the same structural alerts. The conference noted this approach for data-poor situations but did not further discuss it.

\subsubsection{DNA adduct data}

The potential for the use of DNA adduct data for risk assessment has been extensively considered in a workshop of the ILSI Health and Environmental Sciences Institute (HESI) in 2004 (Sander et al., 2005). No consensus emerged from that workshop on the biological significance of low levels of DNA adducts, 
but it identified the need for further experimental data to address this question and for a framework to guide the integration of DNA adduct data into the risk assessment process. The possibilities and limitations associated with the use of such data for risk assessment were illustrated by Gary Williams in his presentation at the conference. As such data are not currently widely used by regulatory bodies except for hazard identification, it was not further discussed.

\subsubsection{Advantages of the MOE approach compared with other approaches}

Since the three documents from ILSI Europe, EFSA and JECFA proposed, or in the case of ILSI Europe and JECFA used, the MOE as the preferred approach for risk assessment advice for substances that are both genotoxic and carcinogenic, the conference discussion was framed in terms of the advantages and limitations of the MOE approach in comparison with other approaches.

The conference considered that the MOE approach offers the following advantages:

- It is a pragmatic approach and has the potential to be explained and understood in a transparent way.

- It lends itself to a narrative presentation of the underlying scientific assumptions.

- It takes account of both carcinogenic potency and exposure which the ALARA approach does not.

- It makes good use of all the available data.

- It does not extrapolate the curve orders of magnitude outside the observable dose-effect range.

- It only has to consider uncertainties in the toxicity data and in the exposure data; it does not have to contend with the uncertainties associated with selection and use of a mathematical model for low dose extrapolation.

- When appropriate human epidemiological data are available, they can be used to calculate MOEs that can be considered instead of, or alongside, those derived from experimental animal data.

- Where uncertainties are identified by the MOE approach, these can indicate what further steps or data may be needed to refine the risk assessment.

- MOEs can be calculated for subsets of the population with different exposures.

- It provides an additional piece of information in a 'weight of evidence' risk assessment.

- At the risk assessment stage, the question of acceptability of risk (a risk management task) can be avoided.

- It can be used to compare and rank substances.

- It can provide guidance on setting priorities for risk management actions. 
- It can provide useful guidance for choosing between different risk management options for a substance.

- It can be used to set targets for risk reduction strategies.

- It can assist the risk manager in decision-making when regulatory limits for a substance are exceeded.

- It can be used by risk managers to distinguish between situations of larger, intermediate and lesser concern and may point to situations of minimal concern.

- It can be used to set priorities for testing and for further research.

- In contrast to linear extrapolation, the MOE approach does not give a risk estimate which may be (mis)interpreted as precise, or as the level of actual risk in the exposed population.

- It can be used to compare the relative risks of exposure to a substance via different routes.

- It can be used to aid decision-making in application of an ALARA/ALARP policy by risk managers.

\subsubsection{Limitations of the MOE approach compared with other approaches}

The conference considered the limitations of the MOE approach:

- It provides a numerical value (a ratio) but, in contrast to linear extrapolation, does not provide a quantitative estimate of risk.

- The abstract nature of a ratio may result in problems of understanding.

- Although it does not define the possible magnitude of the risk, it may be misinterpreted as giving a measure of the risk.

- Good intake/exposure data are critical; the confidence that can be placed in any particular MOE is dependent on the reliability of the exposure/intake assessment.

- Provision of a single value for the MOE could result in over-interpretation of the reliability and applicability of the value.

- As, with all other approaches, it requires a clearly and carefully expressed narrative to provide perspective and context and to explain it to risk managers and consumers.

- Interpretation of the significance of a particular value of an MOE lies on the borders between risk assessment and risk management.

- It does not provide a tool for performing risk/benefit or risk/cost assessments.

\subsubsection{Minimum data sets needed for each approach}

The conference considered the minimum data sets needed for each approach. 


\subsubsection{ALARA}

The minimum data set required for the ALARA approach is:

- Hazard identification data on genotoxicity and carcinogenicity.

\subsubsection{MOE}

It was noted that since in the ideal case dose-response modelling is involved, this requires a certain minimum quantity and quality of animal or human data. The minimum data required for the MOE approach as a whole is:

- An appropriate array of genotoxicity tests with clear evidence of genotoxicity in vitro and/or in vivo.

- Reliable data from animal bioassays and/or human epidemiological studies, with sufficient information to establish carcinogenicity and dose-response relationships; for animal data, this would normally mean a long-term carcinogenicity study conducted according to an internationally recognised guideline in a species showing a clear carcinogenic response.

- If the BMDL is to be determined, at least 3 data points (including controls), with 3 different response levels.

- If the T25 is to be used, one incidence level significantly greater than the controls may suffice.

- Exposure/intake assessment of good quality and representative of the whole diet, for the general population and, where necessary, for subgroups.

- Where there are sources and routes of exposure other than the diet (e.g. dermal, inhalation), these should also be taken into account in the exposure/intake assessment.

\subsubsection{Linear extrapolation}

As for the MOE approach, linear extrapolation includes dose-response modelling, which requires a certain minimum quantity and quality of animal or human data.

The minimum data required for linear extrapolation is the same as for the MOE approach outlined above, but with less emphasis on reliable exposure data. The derivation of the exposure associated with a predetermined risk estimate, such as a 1 in a million risk, does not require any knowledge of human exposure. The end result of linear extrapolation, derived entirely from the biological data, is an upper bound estimate of the dose, expressed on a body weight basis, which causes a specified increase in lifetime risk of cancer. However, exposure data are needed for subsequent comparison of human exposures with the risk estimate.

\subsubsection{Selection of an appropriate reference point from observed data}

Selection of an appropriate reference point, or point of departure, is an important decision in both the MOE and linear extrapolation approaches. The choice should be driven by the extent and quality of the available data. This requires a critical appraisal of the quality of the toxicological data in order to establish the strength of the possible reference point/point of departure. 
Most of those at the conference considered that, if the data are sufficient to enable a BMDL to be determined with confidence, then this is the most appropriate reference point/point of departure. The reason for this choice, in preference to the $\mathrm{BMD}$, is that the $\mathrm{BMDL}$ better reflects the greater uncertainties of lesser amounts of data, since the $95 \%$ confidence interval on the BMD is wider when there are fewer data. A BMDL10 was considered preferable to a BMDL5, since experience shows using data for any one substance, that the various BMDL10 values obtained from different dose-response models are in closer agreement than BMDL5 values. When there is a factor of more than 100 between the BMD and BMDL values, this reflects considerable uncertainty and the BMDL should not be used as a reference point/point of departure. While it may be considered that a $10 \%$ increase in tumour incidence is relatively high value for the benchmark response, the modelling of lower incidences generally results in greater uncertainty.

If the data are insufficient to derive a BMDL10, the conference agreed that use of the T25 was an alternative option. The conference noted that in some cases, for example natural toxicants that are genotoxic and carcinogenic, the database may be so limited that only the ALARA approach would be feasible.

Considering the exposure data that are used to calculate an MOE, the conference suggested that one option would be to work initially with simple worst-case data, and to undertake a more refined exposure/intake assessment if that were necessary for decision-making. This step-wise approach recognises the considerable resources needed for detailed exposure/intake assessments.

\subsubsection{Criteria for choosing between the available approaches}

The conference agreed that the choice of which approach to follow should be case-by-case, based on consideration of the extent of the available data and its quality. The technical criteria for choosing between the various approaches have already been mentioned earlier under consideration of the available tools, the advantages and disadvantages of the various approaches and the minimum data sets required for each approach. In data-poor situations, it may only be possible to give advice to risk managers based on ALARA. In data-rich situations, for example when dose-response modelling gives values for the BMD and its corresponding BMDL that are close, then either the MOE or linear extrapolation approaches are possibilities. The MOE approach is perceived as a more suitable general approach, since it has the advantage of taking exposure scenarios directly into consideration, without the problems associated with providing a numerical risk estimate that may be misinterpreted.

There was support at the conference for using linear extrapolation when adequate human epidemiological data were available, but recognition that this was rarely the case. A decision on whether to use linear extrapolation based on animal bioassay data depends to some extent on whether the risk assessor has confidence in the validity of extrapolating outside the observed dose range to estimate human risk. Participants considered that the validity of linear extrapolation may be more readily supported when data are available on physiologically-based pharmacokinetics in humans and/or animals or when data are available that address mode of action in more detail.

\subsection{Interpretation of the MOE in terms of risk to human health}

\subsubsection{Magnitude of the MOE}


The conference agreed that the MOE should always be accompanied by a narrative to aid interpretation. This should explain its derivation and include a discussion of the quality of the data underpinning the MOE, both those used for hazard characterisation and for the exposure/intake assessment. The nature of any uncertainties in the data and their interpretation should also be addressed. It was emphasised that the basis for decision-making should not be the MOE alone; the MOE was only one component of the overall risk assessment. It was noted that a dialogue between risk assessors and risk managers will be particularly important to facilitate understanding and interpretation of the implications of particular magnitudes of MOEs for human health.

There will be circumstances in which several MOEs for the same substance related to different exposure/intake scenarios are presented and this will need careful explanation. For example, MOEs for different subgroups of the population may differ because of differing exposures. Several MOEs may be given because modelling has been done on more than one individual tumour type, or using more than one point of departure on the dose-response. It should also be recognised that MOEs may change as new data on toxicity and/or exposure become available, or with the implementation of risk reduction strategies.

There were differing views among the conference participants about how to interpret the magnitude of an MOE. Concerns were also expressed about the potential for misuse and misinterpretation of MOEs. There was recognition that MOEs of the same numerical value may need to be viewed differently, for example because of differences in the quality of the carcinogenicity and exposure/intake data. The discussion encompassed not only the significance that might be attached to any particular magnitude of an MOE, but also the extent to which the risk assessor should comment on the magnitude of the MOE, using qualitative terms about risks to human health such as high, low or negligible risk or concern.

It was recognised at the conference that in general terms the higher the $\mathrm{MOE}$, the lower the degree of concern. It was also noted that MOEs based on data from lifetime animal studies are potentially more conservative if actual human exposures are short in duration or sporadic, rather than lifetime. A number of participants had reservations about the rationale for the proposal made by the EFSA Scientific Committee in its Opinion (EFSA, 2005), that in general an MOE of 10,000 or higher, if based on a BMDL10 from an animal study, would be of low concern from a public health point of view and might reasonably be considered as a low priority for risk management actions. It was questioned whether attributing low concern to MOEs at or above 10,000 had been scientifically justified or whether it was more a practical suggestion of where the division between lesser concern and greater concern might lie. It was considered important not to view the figure of 10,000 as some kind of threshold for triggering concern or risk management action. For example, a high MOE should not preclude consideration of taking risk management action, including the application of ALARP.

The EFSA Scientific Committee (EFSA, 2005) considered the figure of 10,000 or higher for an MOE as (in general) being of low concern, because in its view, such an MOE adequately allowed for various uncertainties in the MOE approach, namely:

(i) Species differences and human variability in toxicokinetics (TK) and toxicodynamics (TD).

(ii) Inter-individual human variability in cell cycle control and DNA repair. 
(iii) The fact that the reference point is not a NOAEL.

The EFSA Scientific Committee considered that a 100-fold difference would cover the first area of uncertainty (analogous with the default uncertainty factor for inter-species and inter-individual differences in TK and TD used in conventional risk assessment for toxic effects with a threshold), and that a 100-fold difference would cover the second and third areas of uncertainty. At the conference, there was some agreement that the use of 100 for the first area is scientifically justifiable, but that the second area of uncertainty might actually be covered by the first and that scientific justification for a factor of, say, 10 for the third area of uncertainty was lacking. The conference noted that the JECFA, which had used the MOE approach to reach conclusions on several food contaminants, had not specifically discussed the figure of 10,000 but, in practice, in its discussion of polycyclic aromatic hydrocarbons, it had concluded that MOEs of 10,000 and 25,000 indicated that intakes were of low concern for human health (JECFA, 2005).

There was also a proposal at the conference that the interpretation of the MOEs regarding the potential for public health concern might be better expressed in three or four broad ranges rather than as exact figures. These could then be related to different 'classes of concern'. This would avoid misinterpretation or over-interpretation of individual values. However, defining and justifying the broad ranges would raise exactly the same issues.

It was agreed that there needed to be further discussion on whether and how to assign levels of concern to particular figures or ranges of MOEs. It was also agreed that what might constitute a particular level of concern was ultimately a judgement for risk managers to make.

\subsubsection{Comparison of risks of different chemicals}

There was general agreement that the MOE approach was a suitable method to highlight the comparative risks from different chemicals, provided a consistent approach has been adopted. For example, there should be broadly comparable quality of data available for each chemical on both toxicity and exposure and the same reference point used.

While MOEs can be compared, it is important to recognise that an MOE is not a quantification of risk, but its magnitude can be used to indicate a level of concern, to rank substances, to set priorities and targets for risk management actions, and for making individual lifestyle choices.

Risk managers also needed to be made aware that similar MOEs from different chemicals do not necessarily represent identical risks. Each MOE needs context and interpretation.

\subsection{The way forward and conclusions}

\subsubsection{Technical improvements}

A number of ways of improving the risk assessment of substances that are both genotoxic and carcinogenic were mentioned. These included:

- Generation and integration of data on internal dose. 
- Use of information on toxicokinetics, metabolism and tumour biology to refine the risk assessment.

- Optimisation of study designs to deliver data that are better suited for benchmark dose modelling (e.g. better dose selection, more dose groups with fewer animals per group).

- Integration of intermediate endpoints relevant to genotoxic and carcinogenic modes of action to further refine the risk assessment.

- Better use of biomarkers in humans for exposure and effects.

- Improved intake and occurrence data for chemicals in food, for example by ensuring wide access to a database of European Food Consumption Data (currently under development by EFSA).

\subsubsection{Areas requiring further discussion and development}

A number of key areas requiring further discussion and development emerged from the conference. These were:

(i) There was wide support for the proposition that substances which are genotoxic and carcinogenic should not be deliberately added to food and it was not appropriate for the MOE approach to be used to justify such addition. However, there is a need for further discussion between risk assessors and risk managers on the types of substances in food for which it would be appropriate to apply the MOE approach. For example, should its application be limited to unavoidable contaminants and natural toxicants, or (as proposed in the EFSA opinion) could it be applied in cases where genotoxic and carcinogenic substances have been found in food, irrespective of their origin, and where there is a need for guidance on risks to those who are or have been exposed?

(ii) There is a need for general guidance in a number of areas in order to better harmonise the use of the MOE approach, including:

- The criteria that should be used to assess the adequacy and quality of the data used for risk assessment of genotoxic and carcinogenic substances.

- Guidance on the performance of technical aspects such as dose-response modelling, derivation of reference points, selection of appropriate models, and the use of software.

- Guidance on selection of appropriate exposure scenarios and on the performance of exposure/intake assessments, including probabilistic methods.

- Guidance on how to incorporate mode of action, toxicokinetic and toxicodynamic information into the risk assessment.

- Guidance on the explanation and communication of uncertainties.

(iii) The possibility of developing adjustment factors should be considered. The conference recognised that in many situations, such as a newly discovered contaminant in food, neither the toxicity database nor the exposure information will be optimal. In order to be able to compare MOEs derived from a T25 with those derived from a BMDL10, a scientifically-based adjustment factor may be 
needed. Consideration should also be given to whether adjustment factors can be developed to take account of limitations in the exposure data.

(iv) A critical future discussion concerns the question of weighing the potential health significance of the magnitude of particular MOEs, how to band MOEs with respect to levels of concern and who should be involved in that process. Ultimate decisions on these aspects rest with risk managers. However, all three documents from EFSA, JECFA and ILSI Europe offer the view that risk managers will need guidance from risk assessors in interpreting MOEs. As a basis for future discussions, it was suggested that MOEs could be calculated and then placed in a broader range, to which a level of health concern could then be assigned.

(v) There is a need for transparency. When the MOE approach is used, it should be explained in a transparent manner to risk managers and the public. It was recognised that there was a need to develop risk communication strategies for explaining decisions reached using the MOE approach and that there may be a need to test these strategies and evaluate consumer perceptions about the MOE approach. To this end, it might be helpful to develop example case studies on specific substances, showing how the MOE approach could be used throughout the whole risk analysis process.

(vi) Given that many of the substances in food that are both genotoxic and carcinogenic are inadvertent contaminants or natural toxicants, without "ownership", it would be desirable to have support from national governments for generating the necessary toxicological data.

\subsubsection{Issues requiring further research}

A number of areas requiring further research were also identified:

- Exploration of the consistency between animal and human data for individual substances. For example, it would be useful to compare MOEs obtained from animal bioassay data with those obtained from human epidemiological data in those (few) cases of substances where sufficient data from both are available.

- Use of newer technologies to search for more useful intermediate endpoints on the sequence from covalent binding to DNA through to the development of cancer.

- Continuation of fundamental work on the biological changes underlying the generation and repair of genotoxic and carcinogenic events.

The conference concluded that the MOE approach was a useful and pragmatic option for risk assessment of substances that are both genotoxic and carcinogenic. It has the potential to improve the advice provided to risk managers, since it allows comparison between compounds and prioritisation of risk management actions, especially if the MOE is accompanied by an appropriate narrative explaining inherent uncertainties. Further joint discussions between interested parties should be encouraged in order to promote understanding of the utility and relevance of the MOE approach and further harmonise, at an international level, the application of the MOE approach. These discussions should take place both at the technical level among risk assessors and between risk assessors, risk managers, risk communicators and other stakeholders, such as consumers, industry and the media. 


\section{Acknowledgements}

The organizers of the conference as well as the authors of this report would like to thank all participants (see Appendix) for their valuable contributions to the international conference.

The conference was supported by EFSA, WHO and the Risk Assessment of Genotoxic Carcinogens in Food Task Force of the European Branch of the International Life Sciences Institute (ILSI Europe). Industry members of this ILSI Europe task force are Coca-Cola, Firmenich, Givaudan, Groupe Danone, Nestlé, Procter \& Gamble and Unilever.

\section{References}

Dybing et al., 1997 E. Dybing, T. Sanner, H. Roelfzema, D. Kroese and R.W. Tennant, T25: a simplified carcinogenic potency index: description of the system and study of correlations between carcinogenic potency and species/site specificity and mutagenicity, Pharmacology and Toxicology $\mathbf{8 0}$ (1997), pp. 272-279.

Dybing et al., 2002 E. Dybing, J. Doe, J. Groten, J. Kleiner, J. O’Brien, A. Renwick, J. Schlatter, P. Steinberg, A. Tritscher, R. Walker and M. Younes, Hazard characterisation of chemicals in food and diet: dose-response, mechanisms and extrapolation issues, Food and Chemical Toxicology 40 (2002), pp. 237-282.

Edler et al., 2002 L. Edler, K. Poirier, M. Dourson, J. Kleiner, B. Mileson, H. Nordmann, A. Renwick, W. Slob, K. Walton and G. Würtzen, Mathematical modelling and quantitative methods, Food and Chemical Toxicology 40 (2002), pp. 283-326.

EFSA, 2005 European Food Safety Authority, 2005. Opinion of the Scientific Committee on a request from EFSA related to a harmonised approach for risk assessment of substances which are both genotoxic and carcinogenic (Request No. EFSA-Q-2004-020). Adopted on 18 October 2005. Available from: <http://efsa.eu.int/science/sc_committee/sc_opinions1201/sc_op_ej282_gentox_en3.pdf>.

Gold et al., 1984 L.S. Gold, C.B. Sawyer, R. Magaw, G.M. Backman, M. de Veciana, R. Levinson, N.K. Hooper, W.R. Havender, L. Bernstein, R. Peto, M.C. Pike and B.N. Ames, A carcinogenic potency database of the standardized results of animal bioassays, Environmental Health Perspectives 58 (1984), pp. 9-319.

Gold et al., 1986 L.S. Gold, M. de Veciana, G.M. Backman, R. Magaw, P. Lopipero, M. Smith, M. Blumenthal, R. Levinson, L. Bernstein and B.N. Ames, Chronological supplement to the carcinogenic potency database: standardized results of animal bioassays published through December 1982, Environmental Health Perspectives 67 (1986), pp. 161-200.

Gold et al., 1987 L.S. Gold, T.H. Slone, G. Backman, R. Magaw, M. DaCosta, P. Lopipero, M. Blumenthal and B.N. Ames, Second chronological supplement to the carcinogenic potency database: standardized results of animal bioassays published through December 1984 and by the National Toxicology Program through May 1986, Environmental Health Perspectives 74 (1987), pp. 237-329.

Gold et al., 1989 L.S. Gold, T.H. Slone and L. Bernstein, Summary of carcinogenic potency and positivity for 492 rodent carcinogens in the Carcinogenic Potency Database, Environmental Health Perspectives 79 (1989), pp. 259-272.

Gold et al., 1990 L.S. Gold, T.H. Slone, G.M. Backman, S. Eisenberg, M. DaCosta, M. Wong, N.B. Manley, L. Rohrbach and B.N. Ames, Third chronological supplement to the Carcinogenic Potency Database: standardized results of animal bioassays published through December 1986 and by the National Toxicology Program through June 1987, Environmental Health Perspectives 84 (1990), pp. 215-285. 
Gold et al., 1991 L.S. Gold, T.H. Slone, N.B. Manley, G.B. Garfinkel, E.S. Hudes, L. Rohrbach and B.N. Ames, The carcinogenic potency database: analyses of 4000 chronic animal cancer experiments published in the general literature and by the US National Cancer Institute/National Toxicology Program, Environmental Health Perspectives 96 (1991), pp. 11-15.

Gold et al., 1992 L.S. Gold, T.H. Slone, B.R. Stern, N.B. Manley and B.N. Ames, Rodent carcinogens: setting priorities, Science $\mathbf{2 5 8}$ (1992), pp. 261-265.

Gold et al., 1993 S.L. Gold, N.B. Manley, T.H. Slone, G.B. Garfinkel, L. Rohrbach and B.N. Ames, The fifth plot of the Carcinogenic Potency Database: results of animal bioassays published in the general literature through 1988 and by the National Toxicology Program through 1989, Environmental Health Perspectives 100 (1993), pp. 65-135.

Gold et al., 1995 L.S. Gold, N.B. Manley, T.H. Slone, G.B. Garfinkel, B.N. Ames, L. Rohrbach, B.R. Stern and K. Chow, Sixth plot of the Carcinogenic Potency Database: results of animal bioassays published in the general literature 1989 to 1990 and by the National Toxicology Program 1990 to 1993, Environmental Health Perspectives 103 (1995) (Suppl. 8), pp. 3-123. Abstract-Elsevier BIOBASE | Order Document

Gold et al., 1999 L.S. Gold, N.B. Manley, T.H. Slone and L. Rohrbach, Supplement to the carcinogenic potency database (CPDB): results of animal bioassays published in the general literature in 1993 to 1994 and by the National Toxicology Program in 1995 to 1996, Environmental Health Perspectives 107 (1999) (Suppl. 4), pp. 3-123. Abstract + References in Scopus | Cited By in Scopus

Gray and Collins, 2000 J.W. Gray and C. Collins, Genome changes and gene expression in human solid tumours, Carcinogenesis 21 (2000), pp. 443-452.

IPCS, 2004 International Programme on Chemical Safety, 2004. Principles for modelling low-dose response for risk assessment of chemicals. IPCS Workshop held in 2004. Available from:

<http://www.who.int/ipcs/food/risk_assessment/en/>.

JECFA, 2005 Joint FAO/WHO Expert Committee on Food Additives, 2005. JECFA Sixty-fourth meeting, Rome, 8-17 February 2005. Summary and Conclusions. FAO/WHO. Available from: <ftp://ftp.fao.org/es/esn/jecfa/jecfa64_summary.pdf/>.

Kroes et al., 2004 R. Kroes, A.G. Renwick, M. Cheeseman, J. Kleiner, I. Mangelsdorf, A. Piersma, B. Schilter, J. Schlatter, F. van Schothorst, J.G. Vos and G. Wurtzen, Structure-based thresholds of toxicological concern (TTC): guidance for application to substances present at low levels in the diet, Food and Chemical Toxicology 42 (2004), pp. 65-83.

Loeb and Loeb, 2000 K.R. Loeb and L.A. Loeb, Significance of multiple mutations in cancer, Carcinogenesis 21 (2000), pp. 379-385.

Lutz, 1990 W.K. Lutz, Dose-response relationship and low dose extrapolation in chemical carcinogenesis, Carcinogenesis 11 (1990), pp. 1243-1247.

O'Brien et al., 2006 O'Brien, J., Renwick, A.G., Constable, A., Dybing, E., Müller, D., Schlatter, J., Slob, W., Tueting, W., van Benthem, J., Williams, G.M., Wolfreys, A., 2006. Approaches to the risk assessment of genotoxic carcinogens in food: a critical appraisal. Food and Chemical Toxicology, in press, doi: 10.1016/j.fct.2006.07.004.

Peto et al., 1984 A.E. Peto, M.C. Pike, L. Bernstein, L.S. Gold and B.N. Ames, The TD50: a proposed general convention for the numerical description of the carcinogenic potency of chemicals in chronicexposure animal experiments, Environmental Health Perspectives 58 (1984), pp. 1-8.

Sander et al., 2005 M. Sander, J. Cadet, D.A. Casciano, S.M. Galloway, L.J. Mernett, R.F. Novak, S.D. Pettit, R.J. Preston, J.A. Skare, G.M. Williams, B. Van Houten and B.B. Gollapudi, Proceedings of a workshop on DNA adducts: biological significance and applications to risk assessment, Washington DC, April 13-14, 2004, Toxicology and Applied Pharmacology 208 (2005), pp. 1-20.

Sanner et al., 2001 T. Sanner, E. Dybing, M.I. Willems and E.D. Kroese, A simple method for quantitative risk assessment for non-threshold carcinogens based on the dose descriptor T25, Pharmacology and Toxicology 88 (2001), pp. 331-341. 
Food and Chemical Toxicology Volume 44, Issue 10 , October 2006, Pages 1636-1650

US EPA, 1995 US Environmental Protection Agency, 1995. The use of the benchmark dose approach in health risk assessment. EPA/630/R-94/007. US Environmental Protection Agency, Risk Assessment Forum, Washington, DC.

US EPA, 1996 US Environmental Protection Agency, Proposed guideline for carcinogen risk assessment, Federal Register 6 (1996) (79), pp. 17960-18011.

US EPA, 1999 US Environmental Protection Agency, 1999. Guidelines for carcinogen risk assessment. Draft NCEA-F-0644. US Environmental Protection Agency, Risk Assessment Forum, Washington, DC.

US EPA, 2000a US Environmental Protection Agency, 2000a. BMD Software Documentation. Office of Research and Development. National Center for Environmental Assessment, Research Triangle Park, NC, USA. Available from: <http://cfpub.epa.gov/ncea/cfm/recordisplay.cfm?deid=22507>.

US EPA, 2000b US Environmental Protection Agency, 2000b. Draft Benchmark Dose Technical Guidance Document. External Review Draft. Risk Assessment Forum, Environmental Protection Agency, Washington DC, USA. EPA/630/R-00/001, October 2000. Available from:

<http://cfpub.epa.gov/ncea/cfm/recordisplay.cfm?deid=20871>.

WHO, 1999 World Health Organization, 1999. Principles for the assessment of risk to human health from exposure to chemicals. Environmental Health Criteria No. 210. WHO, Geneva. 